\title{
Modeling fractures as interfaces: a model for Forchheimer fractures
}

\author{
Najla Frih — Jean E. Roberts — Ali Saâda
}

\section{$\mathbf{N}^{\circ} 9999$}

Janvier 2008

Thème NUM 



\title{
RIN RIA
}

\section{Modeling fractures as interfaces: a model for Forchheimer fractures}

\author{
Najla Frih 拥, Jean E. Robert执, Ali Saâda* \\ Thème NUM — Systèmes numériques \\ Projets Estime
}

Rapport de recherche $n^{\circ} 9999$ - Janvier 2008 - 23 pages

\begin{abstract}
In this report we are concerned with modeling single phase flow in a medium with known fractures. In particular we are interested in the case in which the flow rate in the fractures is large enough to make it appropriate to use Forchheimer's law for modeling the flow in the fractures even though the flow in the surrounding domain is such that Darcy's law is adequate. We describe a model in which the fractures are treated as interfaces. We also consider the case of intersecting fractures and the case of nonconforming meshes.
\end{abstract}

Key-words: porous media; Fractures; Forchheimer's law; Mixed finite elements; Domain decomposition; Nonconforming meshes.

\footnotetext{
* ENIT-LAMSIN, BP 37, 1002 Tunis Le Belvédère, Tunisie (Najla.Frih@lamsin.rnu.tn, Ali.Saada@ipein.rnu.tn)

$\dagger$ Inria-Rocquencourt, BP 105, 78153 Le Chesnay Cedex, France (Najla.Frih@inria.fr, Jean.Roberts@inria.fr).
} 


\section{Modélisation de fractures comme des interfaces: un modèle avec écoulement de Forchheimer dans la fracture}

Résumé : Dans ce rapport, nous nous intéressons à la modélisation des écoulements monophasiques dans un milieu poreux fracturé. En particulier, nous supposons que l'écoulement est suffisament rapide dans la fracture pour qu'il soit gouverné par la loi de Forchheimer alors que l'écoulement dans la matrice rocheuse est gouverné par la loi de Darcy. Nous décrivons un modèle où la fracture est considérée comme étant une interface. Nous considérons aussi les cas des fractures qui se croisent et le cas des maillages non-conformes.

Mots-clés : écoulement en milieu poreux; fractures; loi de Forchheimer; éléments finis mixtes; décomposition de domaines; maillages non-conformes. 


\section{Introduction}

Modeling flow in porous media is made difficult by the presence of heterogeneities in the characteristics of the medium. In particular, it is difficult to take into account fractures in a numerical model, because of their width which is very small in comparison to the size of the domain. Yet because their permeability may also differ greatly from that of the surrounding medium, they may have a very important influence on the flow in the medium. They may act as privileged channels for flow or they may act as barriers.

Fractures may occur as networks of very fine fractures, impossible to localize individually, [8. These are taken into account using homogenization techniques or double porosity models, [16], [6]. Here however, we are concerned with larger known fractures. In [1], a model was introduced in which such larger fractures are modeled as $(n-1)$ dimensional interfaces in an $n$ dimensional medium. The fractures were supposed to be of high permeability so the pressure was assumed to be continuous across the fractures. However the flux was not supposed to be continuous as the fluid could flow into and out of as well as along the fractures. A model introduced in [17] generalizes the earlier model so that it can handle both large and small permeability in the fracture. For this model it is no longer assumed that the pressure is continuous across the fracture. In [2], a 3-D model with intersecting fractures was introduced. For all of these models, flow in the fractures as well as in the surrounding medium is governed by Darcy's law. In an earlier article, [13], a model was introduced in which the flow in the fracture is sufficiently rapid that it is governed by a nonlinear law, Forchheimer's law, see [7], [11], [12] and [20]. In the present article we develop the model of [13] in more detail, extending it to the case of intersecting fractures and giving more numerical results. We also show that nonmatching grids may be used for the subdomains and for the fractures.

We mention that others have also treated fractures as interfaces: for Darcy flow, a model introduced by Angot et al. in [3] is based on Robin boundary conditions at the interface and assumes the continuity of the flux across the fracture. A model similar to that of [17] was studied by Flauraud et al. in [10]. For a problem of multiphase flow in a fractured medium, Helmig et. al. in [18], represented the fracture by lower dimensional finite elements.

In Section 2, we describe the different equations governing single phase, incompressible flow in a porous media. In Section 3, we describe a simple model problem with a domain containing a single fracture. Then, in Section 4, the interface model of [13] is derived for this simple problem and in Section 5 we give the discrete formulation along with some numerical results. In Section 6, we extend the simple model problem to a problem with intersecting fractures. In Section 7, we use nonconforming meshes and we show some numerical results with several intersecting fractures.

\section{Equations governing flow in a porous medium}

Flow of a single-phase, incompressible fluid in a porous medium $\Omega$ is governed by the law of mass conservation:

$$
\operatorname{div}(\mathbf{u})=q \text { in } \Omega,
$$

where $q$ is a source term and $\mathbf{u}$ is a volumetric flow rate or velocity. The velocity $\mathbf{u}$ is related to the gradient of the pressure $p$ (in the absence of gravity) by Darcy's law:

$$
\mathbf{u}+K \nabla p=0, \quad \text { in } \Omega,
$$


where $K$ is the permeability of the medium.

Darcy's law is valid for low flow rates for which inertial effects are negligible. For higher flow rates however, the results obtained by Darcy's law do not coincide with experimental results; the flow rate predicted by Darcy's law is too high. Forchheimer's law introduces an inertial term, a quadratic term, which slows down the flow. So, when the flow is sufficiently rapid, Forchheimer's law gives a more accurate relation between the gradient of the pressure and the flow rate. This law is a nonlinear law given by

$$
(1+b|\mathbf{u}|) \mathbf{u}+K \nabla p=0 \text { in } \Omega
$$

where $b$ denotes the Forchheimer constant also called the inertial constant. In some works $b$ is taken to be a tensor, but here we have restricted our attention to the case $b$ is a scalar. For both experimental and theoretical derivations of Forchheimers law see the list of references in the introduction of [15].

\section{Description of a simple model problem}

Suppose that $\Omega$ is a convex domain in $\mathbb{R}^{n}, n=2$ or 3 , and denote by $\Gamma=\partial \Omega$ the boundary of $\Omega$. We suppose that the flow in $\Omega$ is governed by a conservation equation together with Forchheimer's law relating the gradient of the pressure $p$ to the flow velocity $\mathbf{u}$ :

$$
\begin{aligned}
\operatorname{div} \mathbf{u} & =q & & \text { in } \Omega \\
(1+b|\mathbf{u}|) \mathbf{u} & =-K \nabla p & & \text { in } \Omega \\
p & =p_{D} & & \text { on } \Gamma,
\end{aligned}
$$

where $q$ is a source term, $b$ the Forchheimer coefficient, $p_{D}$ the given pressure on the boundary $\Gamma$ and $K$ a diagonal permeability (or hydraulic conductivity) tensor which is supposed to satisfy

$$
0<K_{\min }\|x\|_{0}^{2} \leq(K x, x) \leq K_{\max }\|x\|_{0}^{2}<\infty, \quad \forall x \neq 0 .
$$

For this simple model problem, we suppose (see Figure 1 that the fracture $\Omega_{f}$, a subdomain of $\Omega$, is such that there is a hyperplane $\gamma$ with unit normal vector $\mathbf{n}$ so that

$$
\begin{aligned}
\Omega_{f}= & \{x \in \Omega: x=s+r \mathbf{n} \text { for some } s \in \gamma \cap \Omega \\
& \text { and some } \left.r \text { in the interval }\left(-\frac{d(s)}{2}, \frac{d(s)}{2}\right)\right\},
\end{aligned}
$$

where $d(s)$ denotes the thickness of the fracture at $s \in \gamma$. We also suppose that $\bar{\Omega}_{f}$ separates $\Omega$ into two disjoint, connected subdomains:

$$
\Omega \backslash \bar{\Omega}_{f}=\Omega_{1} \cup \Omega_{2}, \quad \Omega_{1} \cap \Omega_{2}=\emptyset .
$$

We will use the notation $\Gamma_{i}$ for the part of the boundary of $\Omega_{i}$ which lies on $\Gamma, i=1,2, f$

$$
\Gamma_{i}=\partial \Omega_{i} \cap \Gamma, \quad i=1,2, f
$$

and we denote by $\gamma_{i}$ the part of of the boundary of $\Omega_{i}, i=1,2$ which lies on $\partial \Omega_{f}$

$$
\gamma_{i}=\partial \Omega_{i} \cap \partial \Omega_{f} \cap \Omega, \quad i=1,2 .
$$



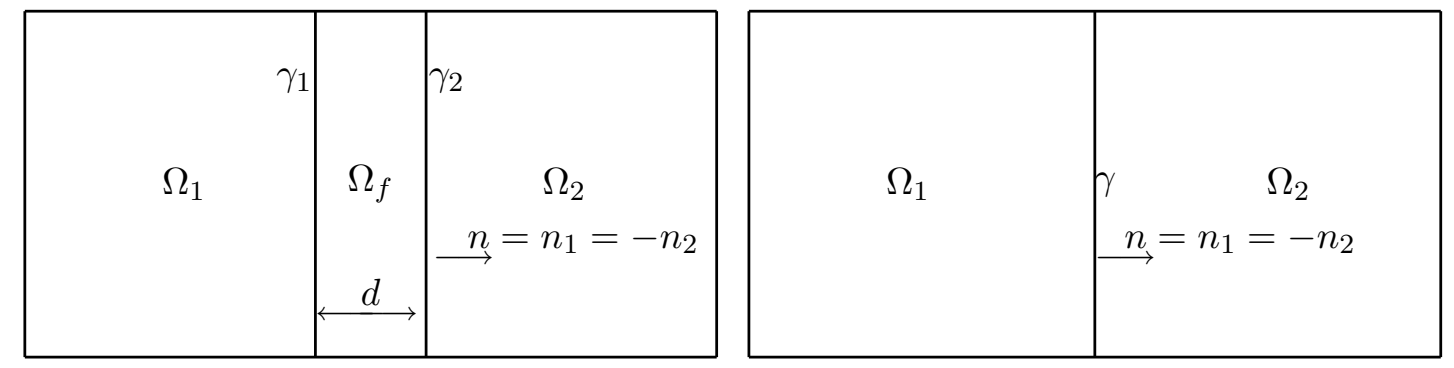

Figure 1: Left: the domain $\Omega$ with the fracture $\Omega_{f}$. Right: the subdomains $\Omega_{1}$ and $\Omega_{2}$ separated by the fracture considered as an interface $\gamma$.

The case of interest for us is that the velocity in the subdomains is small enough for Darcy's law to be sufficient while that in the fracture requires the use of Forchheimer's law. In this case in order to avoid having to solve a nonlinear problem in all of $\Omega$ it would seem appropriate to formulate the problem as a transmission problem using the nonlinear law only in the fracture. If we assume that the flow in the subdomains is governed by Darcy's law and if we denote by $p_{i}, \mathbf{u}_{i}, K_{i}$, and $q_{i}$ the restrictions of $p, \mathbf{u}, K$, and $q$ respectively to $\Omega_{i}, i=1,2, f$, and by $p_{D i}$ the restriction of $p_{D}$ to $\Gamma_{i}, i=1,2, f$ we can rewrite the above problem (4) as a transmission problem:

$$
\begin{aligned}
\operatorname{div} u_{i} & =q_{i} & & \text { in } \Omega_{i}, \quad i=1,2, f \\
\mathbf{u}_{i} & =-K_{i} \nabla p_{i} & & \text { in } \Omega_{i}, \quad i=1,2, \\
\left(1+b\left|\mathbf{u}_{f}\right|\right) \mathbf{u}_{f} & =-K_{f} \nabla p_{f} & & \text { in } \Omega_{f}, \\
p_{i} & =p_{D i} & & \text { on } \Gamma_{i}, \quad i=1,2, f \\
p_{i} & =p_{f} & & \text { on } \gamma_{i}, i=1,2, \\
\mathbf{u}_{i} \cdot \mathbf{n} & =\mathbf{u}_{f} \cdot \mathbf{n} & & \text { on } \gamma_{i}, \quad i=1,2 .
\end{aligned}
$$

The system (5) could then be solved by using a conventional nonoverlapping domain decomposition technique in which $\Omega_{f}$ would be considered simply as a third subdomain. Following [1], [17] and [2], we propose here as in [13] the alternative technique of treating $\Omega_{f}$ not as a subdomain, but as an interface $\gamma$ between the subdomains $\Omega_{1}$ and $\Omega_{2}$ (see Figure 1) on which nonlocal transmission conditions are imposed. The advantage of using domain decomposition for modeling the fractured medium is that one no longer needs to have local refinement around the fracture. However if the fracture is taken to be a subdomain itself this introduces a subdomain much thiner than other subdomains which is not usually desirable for domain decomposition algorithms. Further with a standard domain decomposition approach the elements in the fractures would either have one dimension much smaller than the other dimensions or the grids would be nonconforming requiring the use of mortar elements or other special techniques. By treating the fracture not as a thin subdomain but as an interface this problem is eliminated. An added advantage of the interface approach in the present case with Forchheimer flow in the fracture is that one has only an $(n-1)$ dimensional nonlinear problem to solve instead of an $n$ dimensional one. 


\section{Derivation of the interface model}

The model in which the subdomain $\Omega_{f}$ is replaced by the interface $\gamma$, is obtained by using the technique of averaging across the fracture. For this however, a simplifying hypothesis, that flow in the fracture in the direction normal to the central hyperplane $\gamma$ is much smaller than that in the tangential direction, is used. This hypothesis seems reasonable in light of the very small ratio, width to length of the fracture. Thus we suppose that the flow in the direction normal to the fracture is adequately described by Darcy's law and that in the tangential direction, it is described by Forchheimer's law.

The first step toward deriving the model is to decompose $\mathbf{u}_{f}$ as $\mathbf{u}_{f}=\mathbf{u}_{f, n}+\mathbf{u}_{f, \tau}$ with $\mathbf{u}_{f, n}=\left(\mathbf{u}_{f} \cdot \mathbf{n}\right) \mathbf{n}$ (recall that that $\mathbf{n}=\mathbf{n}_{1}=-\mathbf{n}_{2}$ ), and to introduce the notation $\nabla_{\tau}$ and $\operatorname{div}_{\tau}$ for the tangential gradient and divergence operators and $\nabla_{n}$ and $\operatorname{div}_{n}$ for the normal gradient and divergence operators.

\subsection{Averaging the conservation equation}

With the above notation, the first equation of (5) for $i=f$, may be rewritten as

$$
\operatorname{div}_{n} \mathbf{u}_{f}+\operatorname{div}_{\tau} \mathbf{u}_{f}=q_{f} \quad \text { in } \quad \Omega_{f} .
$$

Integrating in the direction normal to the fracture, one obtains

$$
\mathbf{u}_{f} \cdot \mathbf{n}_{\mid \gamma_{2}}-\mathbf{u}_{f} \cdot \mathbf{n}_{\mid \gamma_{1}}+\operatorname{div}_{\tau} \mathbf{U}_{\gamma}=Q_{\gamma} \quad \text { on } \quad \gamma
$$

where $\mathbf{U}_{\gamma}=\int_{-\frac{d}{2}}^{\frac{d}{2}} \mathbf{u}_{f, \tau} d n$ is the flow rate through a normal cross section of the fracture and $Q_{\gamma}=\int_{-\frac{d}{2}}^{\frac{d}{2}} q_{f} d n$

Then using the continuity of the fluxes across $\gamma_{1}$ and $\gamma_{2}$, the last equation of (5) for $i=1$ and 2 , we may write

$$
\operatorname{div}_{\tau} \mathbf{U}_{\gamma}=Q_{\gamma}+\left(\mathbf{u}_{1} \cdot \mathbf{n}_{1 \mid \gamma_{1}}+\mathbf{u}_{2} \cdot \mathbf{n}_{2 \mid \gamma_{2}}\right) \quad \text { on } \quad \gamma .
$$

This is the conservation equation on $\gamma$ with the additional source term $\mathbf{u}_{1} \cdot \mathbf{n}_{1 \mid \gamma_{1}}+\mathbf{u}_{2} \cdot \mathbf{n}_{2 \mid \gamma_{2}}$ representing the difference between what flows into the fracture and what flows out of the fracture at a given point of $\gamma$.

\subsection{Averaging Forchheimer's law}

With the hypothesis that flow in the fracture in the direction normal to the fracture is described by Darcy's law, the decomposition of the second equation of the system (5) into its tangential direction and the normal direction parts is given by

$$
\begin{aligned}
\left(1+b\left|\mathbf{u}_{f}\right|\right) \mathbf{u}_{f, \tau} & =-K_{f, \tau} \nabla_{\tau} p_{f} \\
\mathbf{u}_{f, n} & =-K_{f, n} \nabla_{n} p_{f} .
\end{aligned}
$$

Integrating the first equation of the system $\left[9\right.$, a) along the line segments $\left(-\frac{d}{2}, \frac{d}{2}\right)$, yields:

$$
\int_{-\frac{d}{2}}^{\frac{d}{2}}\left(1+b\left|\mathbf{u}_{f}\right|\right) \mathbf{u}_{f, \tau} d n=-K_{f, \tau} \nabla_{\tau} \int_{-\frac{d}{2}}^{\frac{d}{2}} p_{f} d n
$$


where we have assumed that $K_{f, \tau}$ is constant along the segments $\left(-\frac{d}{2}, \frac{d}{2}\right)$. We approximate $\left|\mathbf{u}_{f}\right|=\left|\mathbf{u}_{f, \tau}+\mathbf{u}_{f, n}\right|$ by $\left|\mathbf{u}_{f, \tau}\right|\left(1+\frac{1}{2} \frac{\mathbf{u}_{f, n}^{2}}{\mathbf{u}_{f, \tau}^{2}}\right)$.

Then using the hypothesis that $\mathbf{u}_{f, n}$ is very small in comparison to $\mathbf{u}_{f, \tau}$, we obtain the approximation

$$
\left|\mathbf{u}_{f}\right| \simeq\left|\mathbf{u}_{f, \tau}\right| \simeq \frac{\left|\mathbf{U}_{\gamma}\right|}{d}
$$

Replacing $\left|\mathbf{u}_{f}\right|$ by $\frac{\left|\mathbf{U}_{\gamma}\right|}{d}$ in 100 and integrating one obtains

$$
\left(1+\frac{b}{d}\left|\mathbf{U}_{\gamma}\right|\right) \mathbf{U}_{\gamma}=-K_{f, \tau} d \nabla_{\tau} P_{\gamma}
$$

where $P_{\gamma}=\frac{1}{d} \int_{-\frac{d}{2}}^{\frac{d}{2}} p_{f} d n$ is the average pressure along a normal cross section of the fracture.

Equation $(11)^{2}$ is Forchheimer's law in the $(n-1)$ dimensional domain $\gamma$. Together (8) and (11) give a flow equation in $\gamma$ with a source term representing the flow from the subdomains $\Omega_{1}$ and $\Omega_{2}$ into the fracture. The second equation of $(9)$ can now be used to give boundary conditions along $\gamma$ for the subdomains $\Omega_{1}$ and $\Omega_{2}$.

Integrating the second equation of the system $\left[9\right.$ b) along the line segments $\left(-\frac{d}{2}, \frac{d}{2}\right)$, yields:

$$
\int_{-\frac{d}{2}}^{\frac{d}{2}} \mathbf{u}_{f, n}=-K_{f, n} \int_{-\frac{d}{2}}^{\frac{d}{2}} \nabla_{n} p_{f}
$$

where we have assumed that $K_{f, n}$ is constant along the segments $\left(-\frac{d}{2}, \frac{d}{2}\right)$.

Equation $(12)$ is equivalent to

$$
p_{2 \mid \gamma}-p_{1 \mid \gamma}=-\frac{1}{K_{f, n}} \int_{-\frac{d}{2}}^{\frac{d}{2}} \mathbf{u}_{f, n} d n
$$

With the hypotheses that the fracture width $d$ is much smaller than the fracture length, and the high permeability in the fracture, i.e $K_{f, n}$ is large, then we can suppose that the pressure $p$ is continuous through $\gamma$ so that

$$
p_{1 \mid \gamma}=P_{\gamma}=p_{2 \mid \gamma}
$$

\subsection{The strong formulation of the interface model problem}

The interface model for the fracture problem can now be written

$$
\begin{aligned}
\operatorname{div} \mathbf{u}_{i} & =q_{i} & & \text { in } \Omega_{i}, \quad i=1,2 \\
\mathbf{u}_{i} & =-K_{i} \nabla p_{i} & & \text { in } \Omega_{i}, \quad i=1,2 \\
\operatorname{div}_{\tau} \mathbf{U}_{\gamma} & =Q_{\gamma}+\left(\mathbf{u}_{1} \cdot \mathbf{n}-\mathbf{u}_{2} \cdot \mathbf{n}\right) & & \text { on } \gamma \\
\left(1+\frac{b}{d}\left|\mathbf{U}_{\gamma}\right|\right) \mathbf{U}_{\gamma} & =-K_{f, \tau} d \nabla_{\tau} P_{\gamma} & & \text { on } \gamma \\
p_{i} & =p_{D i} & & \text { on } \Gamma_{i}, \quad i=1,2 \\
p_{i} & =P_{\gamma} & & \text { on } \gamma, \quad i=1,2 \\
P_{\gamma} & =P_{D \gamma} & & \text { on } \partial \gamma,
\end{aligned}
$$


where $P_{D \gamma}$ is the average value of $p_{D f}$ along the segment $\left(-\frac{d}{2}, \frac{d}{2}\right)$ in $\Gamma_{f}$.

\section{$5 \quad$ Numerical discretization for the interface model}

\subsection{The discrete formulation of the model problem}

Let $\mathcal{T}_{h, i}$ be a conforming finite element partition of $\bar{\Omega}_{i}, i=1,2$, and suppose that the meshes $\mathcal{T}_{h, i}, i=1,2$ match at the interface $\gamma$ between the subdomains $\Omega_{i}$, i. e. suppose that these partitions are such that the two partitions induced on $\gamma$ by these partitions $\mathcal{T}_{h, i}$ coincide. In other words $\mathcal{T}_{h, 1} \cup \mathcal{T}_{h, 2}$ forms a standard finite element partition of $\Omega$. Then denote by $\mathcal{T}_{h, \gamma}$ the induced mesh on $\gamma$ consisting of all edges of elements of $\mathcal{T}_{h, i}$ that lie on $\gamma$. Then let $\mathcal{T}_{h}=\cup \mathcal{T}_{h, i}$, $i=1,2, \gamma$. Thus $\mathcal{T}_{h}$ consists of both $n$-dimensional elements in $\Omega_{i}$ and $(n-1)$-dimensional elements on $\gamma$.

To define an approximation space for the pressure, let $M_{h, i}, i=1,2$ be the space of piecewise constant functions constant on each element $T$ of $\mathcal{T}_{h, i}$ and let $M_{h, \gamma}$ be the approximation space of piecewise constant functions constant on each element $E$ of $\mathcal{T}_{h, \gamma}$. Then let

$$
M_{h}=M_{h, 1} \times M_{h, 2} \times M_{h, \gamma}
$$

Similarly, to define an approximation space for the velocity, let $\mathbf{W}_{\mathbf{h}, \mathbf{i}}, i=1,2$ be the Raviart-Thomas-Nedelec space of lowest order defined on the $n$-dimensional space $\Omega_{i}$ subordinate to the mesh $\mathcal{T}_{h, i}$, and let $\mathbf{W}_{\mathbf{h}, \gamma}$ be the Raviart-Thomas space of lowest order defined on the $(n-1)$-dimensional interface $\gamma$ subordinate to the mesh $\mathcal{T}_{h, \gamma}$. Then let

$$
\mathbf{W}_{\mathbf{h}}=\mathbf{W}_{\mathbf{h}, 1} \times \mathbf{W}_{\mathbf{h}, 2} \times \mathbf{W}_{\mathbf{h}, \gamma} .
$$

The discrete mixed finite element approximation for the interface problem 14 can now be written as follows:

$$
\begin{aligned}
& \text { Find }\left(\mathbf{u}_{h, 1}, \mathbf{u}_{h, 2}, \mathbf{U}_{h, \gamma}\right) \in \mathbf{W}_{\mathbf{h}} \text { and }\left(p_{h, 1}, p_{h, 2}, P_{h, \gamma}\right) \in M_{h} \\
& \text { such that for all }\left(\mathbf{v}_{h, 1}, \mathbf{v}_{h, 2}, \mathbf{V}_{h, \gamma}\right) \in \mathbf{W}_{h} \text { and }\left(r_{h, 1}, r_{h, 2}, r_{h, \gamma}\right) \in M_{h} \\
& \int_{\Omega_{i}} K_{i}^{-1} \mathbf{u}_{h, i} \cdot \mathbf{v}_{h, i}-\int_{\Omega_{i}} p_{h, i} \operatorname{div} \mathbf{v}_{h, i}=-\int_{\gamma} P_{h, \gamma} \mathbf{v}_{h, i} \cdot \mathbf{n}_{i}-\int_{\Gamma_{i}} p_{D i} \mathbf{v}_{h, i} \cdot \mathbf{n}_{i}, i=1,2 \\
& \left(\mathcal{P}_{h}\right) \int_{\Omega_{i}} \operatorname{div} \mathbf{u}_{h, i} r_{h, i}=\int_{\Omega_{i}} q_{i} r_{h, i}, \quad i=1,2 \\
& \int_{\gamma}\left(d K_{f, \tau}\right)^{-1}\left(1+\frac{b}{d}\left|\mathbf{U}_{h, \gamma}\right|\right) \mathbf{U}_{h, \gamma} \cdot \mathbf{V}_{h, \gamma}-\int_{\gamma} P_{h, \gamma} \operatorname{div}_{\tau} \mathbf{V}_{h, \gamma}=-\int_{\partial \gamma} P_{D \gamma} \mathbf{V}_{h, \gamma} \cdot \mathbf{n}_{\gamma} \\
& \int_{\gamma} \operatorname{div}_{\tau} \mathbf{U}_{h, \gamma} r_{h, \gamma}=\int_{\gamma} Q_{\gamma} r_{h, \gamma}+\int_{\gamma}\left(\mathbf{u}_{h, 1} \cdot \mathbf{n}_{1}+\mathbf{u}_{h, 2} \cdot \mathbf{n}_{2}\right) r_{h, \gamma} .
\end{aligned}
$$

Following [1, 2, 17], we use a domain decomposition type method [9] to solve the discrete problem $\left(\mathcal{P}_{h}\right)$. In this way, the problem 115 is reduced to a problem only on the interface $\gamma$. For $i=1,2, \mathbf{u}_{h, i}$ and $p_{h, i}$ are decomposed into two parts,

$$
\mathbf{u}_{h, i}=\mathbf{u}_{h, i}^{0}+\mathbf{u}_{h, i}^{*}, p_{h, i}=p_{h, i}^{0}+p_{h, i}^{*} .
$$


For each $i$, we use the Steklov-Poincaré operator

$$
\begin{aligned}
\mathcal{S}_{i}: M_{h, \gamma} & \longrightarrow M_{h, \gamma} \\
r_{h, \gamma} & \mapsto-\mathbf{u}_{h, i}^{0} \cdot \mathbf{n}_{i}
\end{aligned}
$$

which associates to an element $r_{h, \gamma} \in M_{h, \gamma}$ the element $-\mathbf{u}_{h, i}^{0} \cdot \mathbf{n}_{i} \in M_{h, \gamma}$ where $\left(\mathbf{u}_{h, i}^{0}, p_{h, i}^{0}\right) \in$ $\mathbf{W}_{\mathbf{h}, \mathbf{i}} \times M_{h, i}$ is the solution of the problem

Find $\left(\mathbf{u}_{h, i}^{0}, p_{h, i}^{0}\right) \in \mathbf{W}_{\mathbf{h}, \mathbf{i}} \times M_{h, i}$ such that

$$
\begin{gathered}
\left(\mathcal{P}_{i}^{0}\right) \quad \int_{\Omega_{i}} K_{i}^{-1} \mathbf{u}_{h, i}^{0} \cdot \mathbf{v}_{h, i}-\int_{\Omega_{i}} p_{h, i}^{0} \operatorname{div} \mathbf{v}_{h, i}=-\int_{\gamma} r_{h, \gamma} \mathbf{v}_{h, i} \cdot \mathbf{n}_{i} \quad \forall \mathbf{v}_{h, i} \in \mathbf{W}_{\mathbf{h}, \mathbf{i}} \\
\int_{\Omega_{i}} \operatorname{div} \mathbf{u}_{h, i}^{0} r_{h, i}=0 \quad \forall r_{h, i} \in M_{h, i} .
\end{gathered}
$$

We also use the notation $\chi_{i}=\mathbf{u}_{h, i}^{*} \cdot \mathbf{n}_{i}, i=1,2$, where $\left(\mathbf{u}_{h, i}^{*}, p_{h, i}^{*}\right) \in \mathbf{W}_{\mathbf{h}, \mathbf{i}} \times M_{h, i}$ is the solution of the problem

Find $\left(\mathbf{u}_{h, i}^{*}, p_{h, i}^{*}\right) \in \mathbf{W}_{\mathbf{h}, \mathbf{i}} \times M_{h, i}$ such that

$$
\begin{aligned}
&\left(\mathcal{P}_{i}^{*}\right) \quad \int_{\Omega_{i}} K_{i}^{-1} u_{h, i}^{*} \cdot \mathbf{v}_{h, i}-\int_{\Omega_{i}} p_{h, i}^{*} \operatorname{div} \mathbf{v}_{h, i}=-\int_{\Gamma_{i}} p_{D i} \mathbf{v}_{h, i} \cdot \mathbf{n}_{i} \quad \forall \mathbf{v}_{h, i} \in \mathbf{W}_{\mathbf{h}, \mathbf{i}} \\
& \int_{\Omega_{i}} \operatorname{div} \mathbf{u}_{h, i}^{*} r_{h, i}=\int_{\Omega_{i}} q_{i} r_{h, i} \quad \forall r_{h, i} \in M_{h, i} .
\end{aligned}
$$

Then if $\left(p_{h, 1}, p_{h, 2}, P_{h, \gamma}\right)$ with $\left(\mathbf{u}_{h, 1}, \mathbf{u}_{h, 2}, \mathbf{U}_{h, \gamma}\right)$ is the solution of $\left(\mathcal{P}_{h}\right),\left(\mathbf{u}_{h, i}^{0}, p_{h, i}^{0}\right)$ is the solution of $\left(\mathcal{P}_{i}^{0}\right)$ with $r_{h, \gamma}=P_{h, \gamma}$ and $\left(\mathbf{u}_{h, i}^{*}, p_{h, i}^{*}\right)$ is the solution of $\left(\mathcal{P}_{i}^{*}\right)$ one has

$$
p_{h, i}=p_{h, i}^{0}+p_{h, i}^{*} \quad \text { and } \quad \mathbf{u}_{h, i}=\mathbf{u}_{h, i}^{0}+\mathbf{u}_{h, i}^{*} .
$$

Thus $\mathbf{u}_{h, i} \cdot \mathbf{n}_{i}=\mathcal{S}_{i}\left(P_{h, \gamma}\right)+\chi_{i}$, and $\left(\mathbf{U}_{h, \gamma}, P_{h, \gamma}\right)$ is the solution of the following problem:

Find $\left(\mathbf{U}_{h, \gamma}, P_{h, \gamma}\right) \in \mathbf{W}_{\mathbf{h}, \gamma} \times M_{h, \gamma}$

such that for all $r_{h, \gamma} \in M_{h, \gamma}$ and $\mathbf{V}_{h, \gamma} \in \mathbf{W}_{\mathbf{h}, \gamma}$

$$
\begin{array}{r}
\left(\mathcal{P}_{\gamma}\right) \quad \int_{\gamma}\left(d K_{f, \tau}\right)^{-1}\left(1+\frac{b}{d}\left|\mathbf{U}_{h, \gamma}\right|\right) \mathbf{U}_{h, \gamma} \cdot \mathbf{V}_{h, \gamma}-\int_{\gamma} P_{h, \gamma} \operatorname{div}_{\tau} \mathbf{V}_{h, \gamma}=-\int_{\partial \gamma} p_{D \gamma} \mathbf{V}_{h, \gamma} \cdot \mathbf{n}_{f} \\
\int_{\gamma} \operatorname{div}_{\tau} \mathbf{U}_{h, \gamma} r_{h, \gamma}+\int_{\gamma}\left(\mathcal{S}_{1}\left(P_{h, \gamma}\right)+\mathcal{S}_{2}\left(P_{h, \gamma}\right)\right) r_{h, \gamma}=\int_{\gamma} Q_{\gamma} r_{h, \gamma}+\int_{\gamma}\left(\chi_{1}+\chi_{2}\right) r_{h, \gamma} .
\end{array}
$$

This problem is nonlinear but it is only an (n-1) dimensional nonlinear problem, and it can be solved by using a fixed point iteration method or a quasi-Newton method. In either case the iteration can be initialized with the solution for Darcy flow in the fracture. The solutions $\left(\mathbf{u}_{h, i}, p_{h, i}\right)$ in the subdomains can then be calculated using (18).

\subsection{Some 2-D numerical results}

A first numerical result for this type of problem is to obtain a good correspondence between the solution obtained by the interface problem and a reference solution. We obtain a reference solution by using the model problem where the fracture is considered as a third subdomain and using a mixed finite element method. 


\subsubsection{A simple test case}

In this section numerical results obtained with the interface model will be compared with those obtained using a standard model with 2-D fracture. The results are obtained for a simple model problem: the subdomains $\Omega_{1}$ and $\Omega_{2}$ are both squares of unit length and width, and the fracture $\Omega_{f}$ separating the subdomains is of unit length and of width $d=0.01$. The permeability in each of the subdomains is assumed to be constant and equal to $10^{-9}$, and the fracture is assumed to be of much higher (also constant) permeability, $K_{f}=10^{-6}$. The Forchheimer coefficient $b$ is taken to be 10. The upper and lower boundaries of the the two subdomains are assumed to be impermeable, and there is a pressure drop from right to left of $10^{6}$. The same pressure drop from top to bottom of the fracture is imposed. See Figure 2.

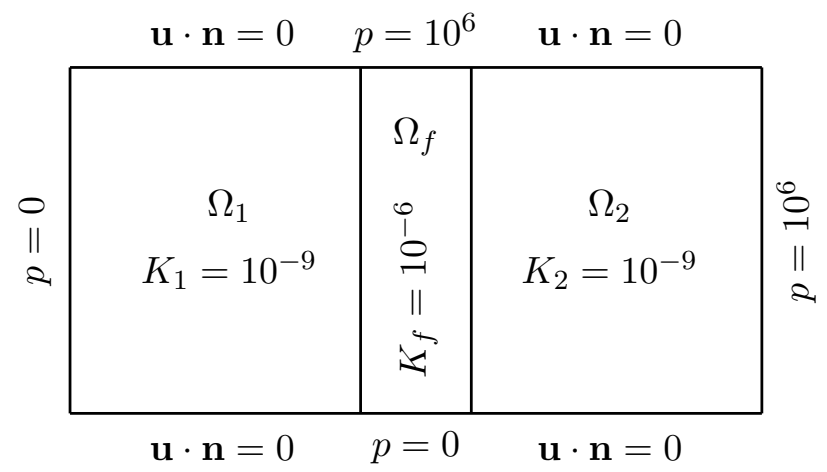

Figure 2: Test-case

To compare the different results, one can see in Figure 3 the reference pressure and also the reference velocity, both obtained by using the standard model (model with 2-D fracture). In Figure 4, those obtained by using the interface model are given. In both cases a 15 by 15 grid was used for each of the subdomains $\Omega_{1}$ and $\Omega_{2}$, and in the case of the reference model a 15 by 15 grid was also used for the fracture domain $\Omega_{f}$. As we can see, these results show good agreement between the solutions obtained by the two models.
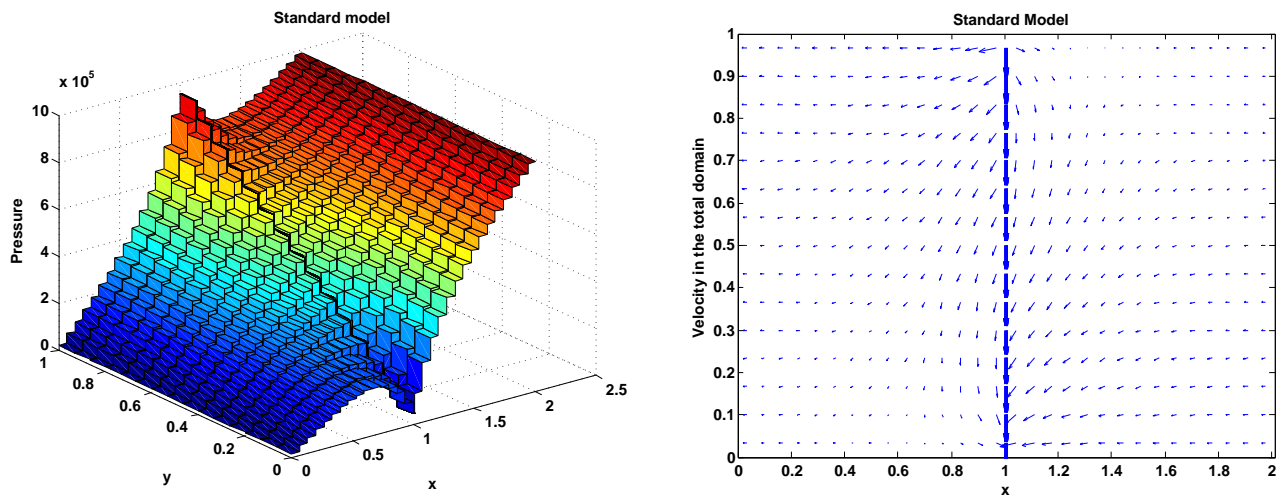

Figure 3: Reference pressure (left) and reference velocity (right) given by the standard model 

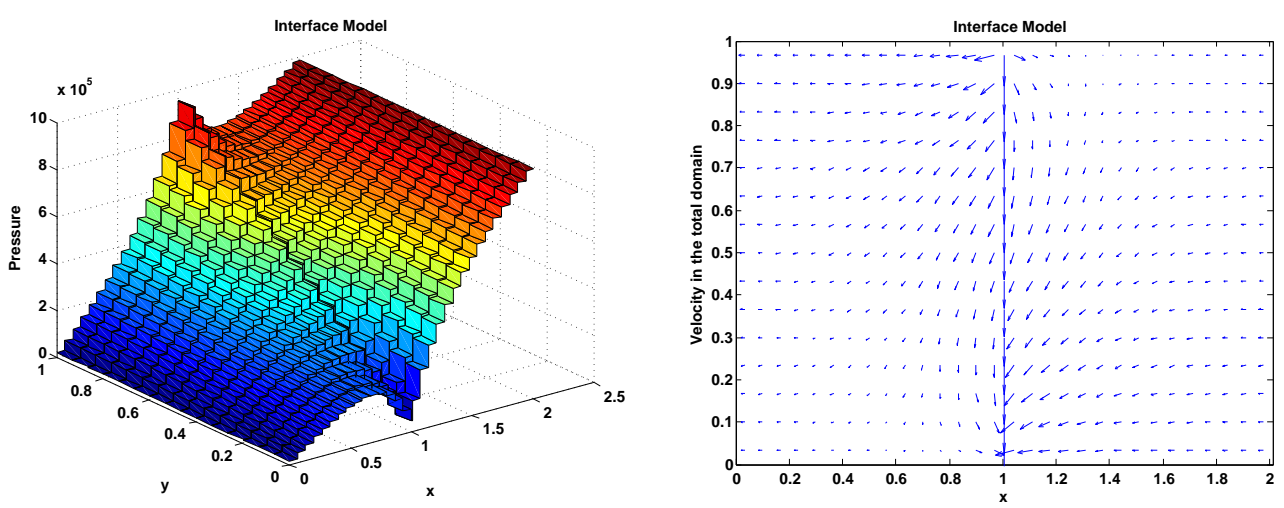

Figure 4: Numerical pressure (left) and numerical velocity (right) given by the interface model

\subsubsection{Comparison between Darcy and Forchheimer velocity in the fracture}

In the experiment above the data were chosen so that the Forchheimer term would have a non negligible effect. Here a comparison between the Darcy and Forchheimer velocities in the fracture is given. The same data as in the above experiment are used only in one case the Forchheimer term in the fracture is omitted.

In Figure 5 the tangential Darcy velocity (which for this simple 2-D model is just a scalar) is represented in blue while the Forchheimer velocity is given by the red curve. On the left, the velocity calculated using the standard model along a central vertical section is shown, and on the right is the velocity obtained using the interface model.
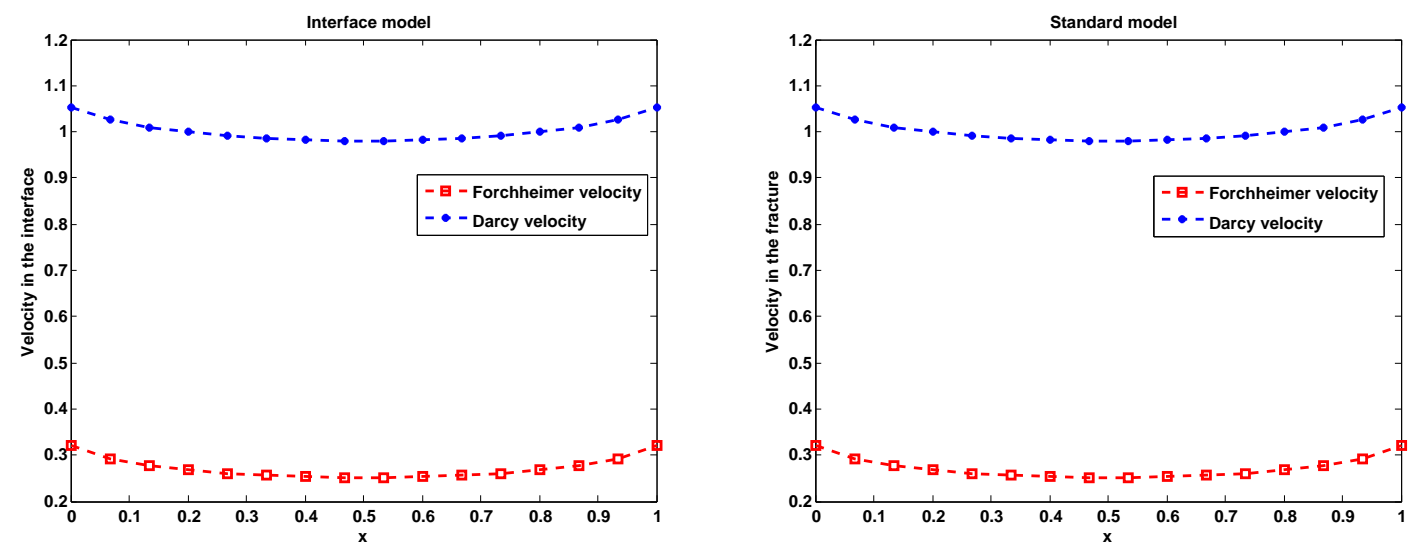

Figure 5: Darcy and Forchheimer velocity in the fracture given both by the interface model (left) and the standard model (right).

A good agreement between the results obtained by the standard model and the interface model can be observed and in addition, the difference between the Forchheimer velocity and 
the Darcy velocity can be seen. The Forchheimer velocity is of much smaller magnitude than the Darcy velocity because of the large inertial coefficient.

Indeed, in this example the Forchheimer coefficient $b$ was taken to be extremely large to show that the model is valid even when the nonlinear term is very important. For more physically correct values of $b$, the percent discrease in the magnitude of the velocity when the Forchheimer law is used instead of simply the Darcy law is much smaller. To illustrate this, we have shown in Figure 6, the average percent decrease in the velocity due to the Forchheimer term for different values of the Forchheimer coefficient $b$. The percentage calculated using the interface model is represented in blue and that obtained using the standard model is given in red.

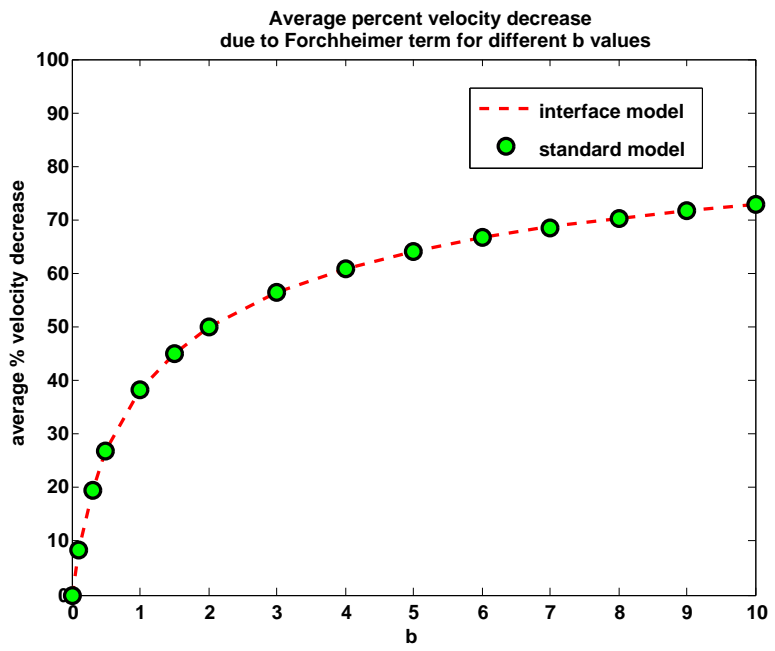

Figure 6: Average precent velocity decrease due to Forchheimer term for different values of the Forchheimer coefficient $b$. 


\subsubsection{Numerical study of the error}

The object of this paragraph is to obtain a quantitative appreciation of the error made when using the interface model. A relative $L^{2}$ error in the pressure in the subdomains is calculated. A reference solution $P_{\eta}^{*}$ is obtained using the standard model with 2-D fracture, using mixed finite elements on a regular fine mesh $\mathcal{T}_{\eta}$ of square elements of side length $\eta$, with $\eta=1 / 192$ in the subdomains $\Omega_{1}$ and $\Omega_{2}$. Approximate solutions $P_{h}$ for different values of $h$ are calculated using the interface model with a uniform mesh of square elements of side length $h$ in each subdomain and a 1-D mesh in the fracture with elements of length $h$.

The discretization parameter $h$ for each of the calculations below is chosen such that the fine mesh in the subdomains is a refinement of the mesh of size $h$. Then a projection $\Pi_{\eta} P_{h}$ of each approximate solution $P_{h}$ onto the fine mesh is obtained in the obvious way. Then the relative $L^{2}$ error in the pressure is given by

$$
\left\|P_{h}-P_{\eta}^{*}\right\|_{L_{r e l}^{2}(\Omega)}^{2}=\frac{\sum_{C_{\eta} \in \mathcal{T}_{\eta}}\left(\Pi_{\eta} P_{h}-P_{\eta}^{*}\right)^{2}\left|C_{\eta}\right|}{\sum_{C_{\eta} \in \mathcal{T}_{\eta}}\left(P_{\eta}^{*}\right)^{2}\left|C_{\eta}\right|}
$$

where $\left|C_{\eta}\right|$ is the measure of the cell $C_{\eta}$.

In these experiments there are two sources of error, the usual numerical error dependent upon the discretisation parameter $h$ and an error due to the interface model dependent upon the width $d$ of the fracture.

In Figure 7, on the left, the log of the relative $L^{2}$ pressure error in the total domain is plotted as a function of the $\log$ of the fracture width $d$. Each curve corresponds to a different value of the mesh size $h: h=1 / 6, h=1 / 12, h=1 / 24, h=1 / 48, h=1 / 96, h=1 / 192$. On the right, the log of the relative $L^{2}$ pressure error in the total domain is plotted as a function of the $\log$ of $1 / h$, the inverse of the mesh size, for a constant fracture width $d=0.01$.

In the first five curves on the left there is no decrease in the error when the fracture width diminishes because the error due to the model is dominated by the error due to the numerical discretization. Only in the sixth curve with $h=1 / 192$ can we see that the error diminishes with decreasing $d$ just until the numerical discretization error again becomes dominant. In the curve on the right, as expected, the error decreases as $1 / h$ increases. The large drop in the error between $1 / h=96$ and $1 / h=192$ is due to the fact that the reference solution is not an analytic solution but a solution obtained with a mesh for which $h=1 / 192$.

\section{A model with intersecting fractures}

\subsection{Description of the problem}

For this model problem, as earlier, $\Omega$ is supposed to be a convex domain in $\mathbb{R}^{n}, n=2$ or 3 , with boundary $\Gamma=\partial \Omega$ decomposed into a Dirichlet part $\Gamma_{D}$ and a Neumann part $\Gamma_{N}$. For simplicity, we suppose that there are three fractures $\gamma_{k}, k=1,2,3$, dividing $\bar{\Omega}$ into three 

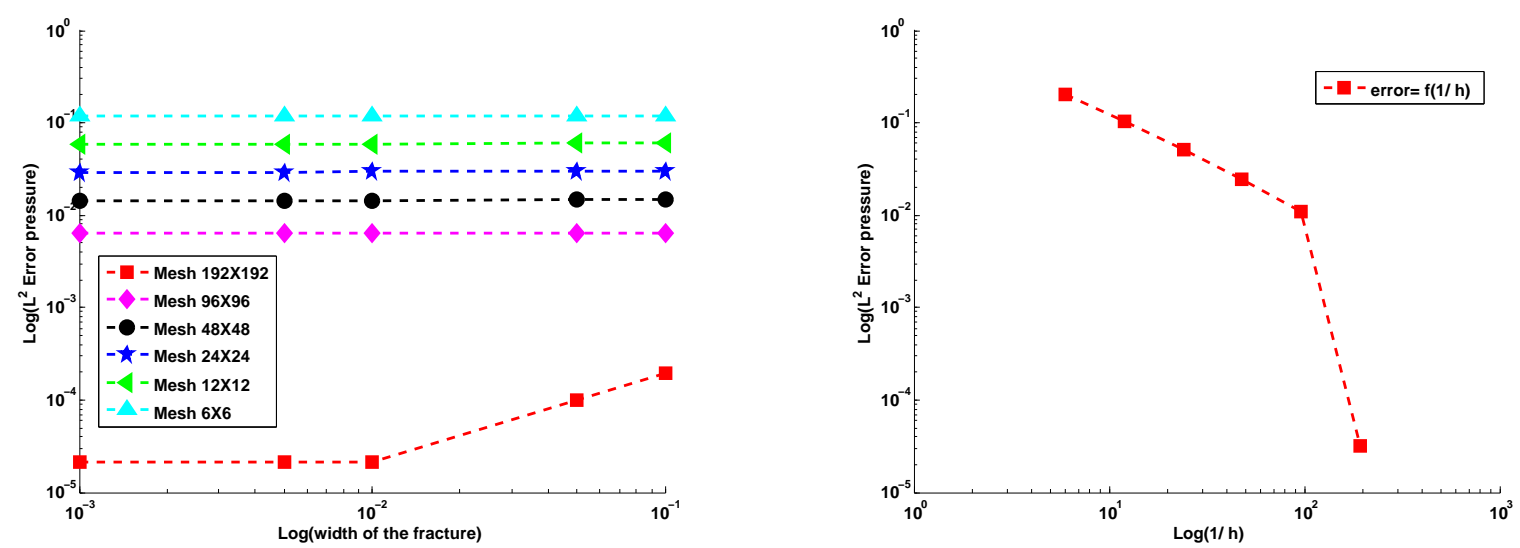

Figure 7: Left: the relative $L^{2}$ error in the pressure in the total domain as a function of the fracture width $d$ for different values of the mesh size: $h=1 / 6, h=1 / 12, h=1 / 24, h=1 / 48$, $h=1 / 96, h=1 / 192$. Right: the relative $L^{2}$ error variation in the total domain as a function of $1 / h$ for a constant fracture width $d=0.01$.

subdomains $\Omega_{i}, i=1,2,3$,

$$
\bar{\Omega}=\bigcup_{i=1}^{3} \bar{\Omega}_{i}, \quad \Omega_{i} \cap \Omega_{j}=\emptyset \quad \text { and } \quad \bar{\Omega}_{i} \cap \bar{\Omega}_{j}=\bar{\gamma}_{k} \quad \forall i, j, k \text { with } i \neq j \neq k \neq i,
$$

in such a way that the intersection of each pair of distinct fractures is the same set $T$ :

$$
\bar{\gamma}_{1} \cap \bar{\gamma}_{2}=\bar{\gamma}_{1} \cap \bar{\gamma}_{3}=\bar{\gamma}_{2} \cap \bar{\gamma}_{3}=T \text {. }
$$

The intersection $T$ is thus a point in the $2-\mathrm{D}$ case and a segment in the 3 -D case. See Figure 8.

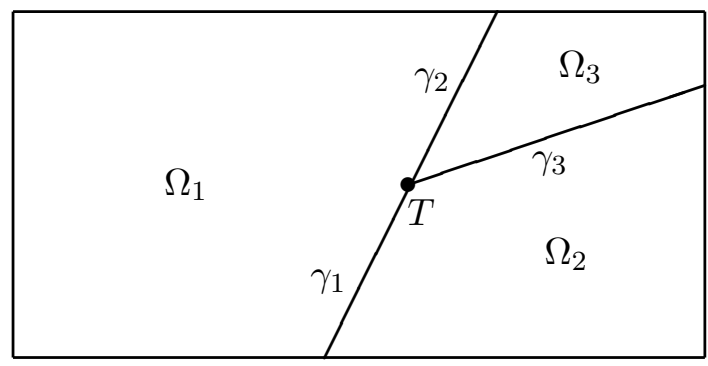

Figure 8: A domain with intersecting fractures

As before, in each subdomain $\Omega_{i}, i=1,2,3$, we have the law of mass conservation together with Darcy's law and the boundary conditions on the exterior boundary:

$$
\begin{aligned}
\operatorname{div} \mathbf{u}_{i} & =q_{i} & & \text { in } \Omega_{i} \\
\mathbf{u}_{i} & =-K_{i} \nabla p_{i} & & \text { in } \Omega_{i} \\
p_{i} & =p_{D i} & & \text { on } \partial \Omega \cap \Gamma_{D} \\
\mathbf{u}_{i} \cdot \mathbf{n}_{i} & =0 & & \text { on } \partial \Omega \cap \Gamma_{N} .
\end{aligned}
$$


Also as before, we assume that the pressure is continuous across the fracture interfaces $\gamma_{k}$ :

$$
p_{i}=P_{\gamma_{k}}=p_{j} \quad \text { on } \quad \gamma_{k}, \quad i \neq j \neq k \neq i,
$$

where $P_{\gamma_{k}}$ is the pressure on the fracture $\gamma_{k}$. In each fracture we have the law of mass conservation together with Forchheimer's law and the boundary conditions on the exterior boundary:

$$
\begin{aligned}
\operatorname{div}_{\tau} \mathbf{U}_{\gamma_{k}} & =Q_{\gamma_{k}}+\left(\mathbf{u}_{i} \cdot \mathbf{n}_{i}+\mathbf{u}_{j} \cdot \mathbf{n}_{j}\right) & & \text { on } \gamma_{k} \\
\left(1+\frac{b_{\gamma_{k}}}{d_{\gamma_{k}}}\left|\mathbf{U}_{\gamma_{k}}\right|\right) \mathbf{U}_{\gamma_{k}} & =-d_{\gamma_{k}} K_{\gamma_{k}} \nabla_{\tau} P_{\gamma_{k}} & & \text { on } \gamma_{k} \\
P_{\gamma_{k}} & =P_{D \gamma_{k}} & & \text { on } \partial \gamma_{k} \cap \Gamma,
\end{aligned}
$$

where $\mathbf{U}_{\gamma_{k}}, Q_{\gamma_{k}}, K_{\gamma_{k}}, b_{\gamma_{k}}$ and $d_{\gamma_{k}}$ are respectively the pressure, the flow velocity, the source term, the permeability tensor, the Forchheimer coefficient and the width of the fracture for $\gamma_{k}$, and $P_{D \gamma_{k}}$ is the average value of $p_{D}$ on $\partial \gamma_{k} \cap \Gamma$.

On the intersection $T$ of the fractures, following [1] and [2], we impose the continuity of the pressure and also the continuity of the flux:

$$
\begin{aligned}
P_{\gamma_{1}}=P_{\gamma_{2}}=P_{\gamma_{3}} & \text { on } T \\
\mathbf{U}_{\gamma_{1}} \cdot \nu_{\gamma_{1}}+\mathbf{U}_{\gamma_{2}} \cdot \nu_{\gamma_{2}}+\mathbf{U}_{\gamma_{3}} \cdot \nu_{\gamma_{3}}=0 & \text { on } T,
\end{aligned}
$$

where $\nu_{\gamma_{k}}$ is the exterior normal to $\gamma_{k}$ on $\partial \gamma_{k}, k=1,2,3$.

\subsection{Numerical discretization}

As in the case of a single simple fracture, to obtain a numerical solution of the system 21 , ..., 24, we use mixed finite elements of dimension $n$ in the subdomains and mixed finite elements of dimension $(n-1)$ in the fractures. Thus as before the pressure is approximated in each element (in dimension $n$ and in dimension $n-1$ ) by a constant, and the velocity is approximated in the space of lowest order Raviart-Thomas-Nedelec elements. However, in addition to the unknowns associated with these spaces there are scalar unknowns associated with the the intersection $T$. In the case $\Omega \subset \mathbb{R}^{2}$ so that $T$ is simply a point this amounts to one unknown which is a multiplier for enforcing continuity of the pressure at the fracture intersection. In the case $\Omega \subset \mathbb{R}^{3}$ so that $T$ is a segment, there is a naturally induced mesh on $T$ from each fracture having $T$ as part of its boundary, and as we have assumed here that the meshes are compatible, i. e. the union of the meshes for the subdomains forms a conforming mesh on all $\Omega$, the meshes induced on $T$ from the different fractures having $T$ as part of their boundaries are all the same. Thus there is one additional unknown for each element of this mesh. 
Again as the nonlinearity of the problem is associated only with the fractures, domain decomposition techniques can be used to reduced the problem to a problem posed only on the fractures. The resulting nonlinear system can now be written as follows:

$$
\left[\begin{array}{ccccccc}
A_{1}\left(U_{\gamma_{1}}\right) & -B_{1}^{\top} & 0 & 0 & 0 & 0 & M_{1}^{\top} \\
B_{1} & S_{1,1} & 0 & S_{1,2} & 0 & S_{1,3} & 0 \\
0 & 0 & A_{2}\left(U_{\gamma_{2}}\right) & -B_{2}^{\top} & 0 & 0 & M_{2}^{\top} \\
0 & S_{2,1} & B_{2} & S_{2,2} & 0 & S_{2,3} & 0 \\
0 & 0 & 0 & 0 & A_{3}\left(U_{\gamma_{3}}\right) & -B_{3}^{\top} & M_{3}^{\top} \\
0 & S_{3,1} & 0 & S_{3,2} & B_{3} & S_{3,3} & 0 \\
M_{1} & 0 & M_{2} & 0 & M_{3} & 0 & M_{T}
\end{array}\right]\left[\begin{array}{c}
U_{\gamma_{1}} \\
P_{\gamma_{1}} \\
U_{\gamma_{2}} \\
P_{\gamma_{2}} \\
U_{\gamma_{3}} \\
P_{\gamma_{3}} \\
P_{T}
\end{array}\right]=\left[\begin{array}{c}
* \\
* \\
* \\
* \\
* \\
* \\
0
\end{array}\right]
$$

where $U_{\gamma_{k}}$ and $P_{\gamma_{k}}$ for $k=1,2,3$, are the velocity and the pressure respectively on the interface $\gamma_{k}$ and $P_{T}$ the trace of the pressure on the intersection $T$ of the fractures. The matricies $A_{k}\left(U_{\gamma_{k}}\right)$ and $B_{k}$, represent the mixed finite element matricies for the flow equations in the fracture $\gamma_{k}, k=1,2,3$. The matrix $S_{i, j}, i, j=1,2,3$, represents the domain decomposition matrix associated with the Steklov-Poincaré operator taking into account the values of the pressure on the fracture $\gamma_{i}$ and returning the values of the flux on the fracture $\gamma_{j}$. The matrix $M_{i}$ with $i=\{1,2,3, T\}$ are the matrices associated with the equations of the continuity of the pressure and the continuity of the flux on the intersection of the fractures.

\subsection{A numerical result for the case of intersecting fractures}

In this experiment the domain $\Omega \subset \mathbb{R}^{2}$ is divided into 4 subdomains $\Omega_{i}, i=1, \ldots, 4$ which are separated by 4 fractures $\gamma_{k}, k=1, \ldots, 4$. These fractures intersect at the point $T$; see Figure 9. The width $d_{\gamma_{k}}$ is the same in all fractures and is equal to 0.01 . The permeability in each of the subdomains is assumed to be constant and equal to $10^{-9}$, and the permeability in each fracture is assumed to be much higher (also constant) $K_{\gamma_{k}}=10^{-6}$. The Forchheimer coefficient $b_{\gamma_{k}}$ is the same in all fractures and is taken to be 10. The upper and lower exterior boundaries of the subdomains are assumed to be impermeable, and there is a pressure drop from right to left of $10^{6}$. A pressure of $10^{6}$ is imposed at the ends of the fractures lying on the upper and right-hand sides of the domain and a pressure of 0 is imposed on the ends of the fractures on the left-hand and lower sides of the domain.

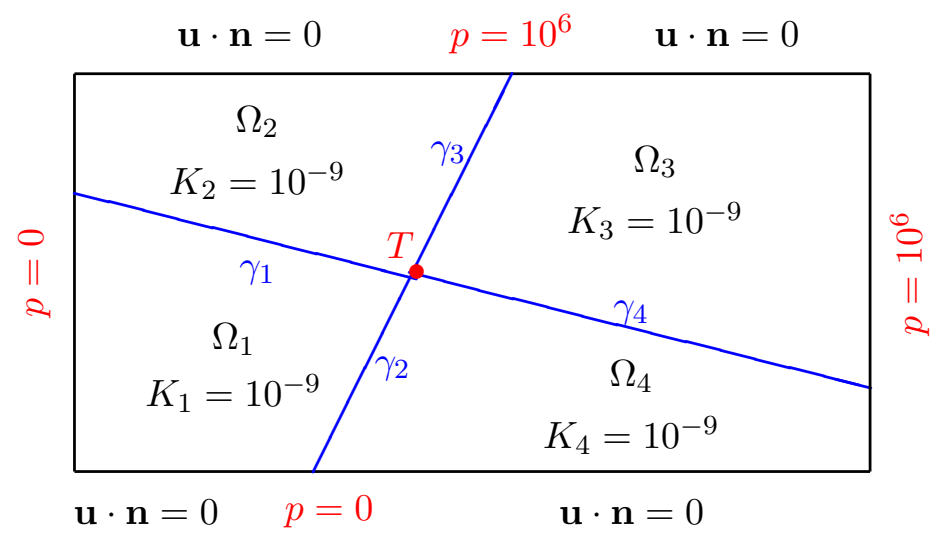

Figure 9: A test-case 
In Figure 10 the conforming mesh used for this test case is shown. In Figure 11 the calculated pressure is shown on the left and the calculated velocity on the right.

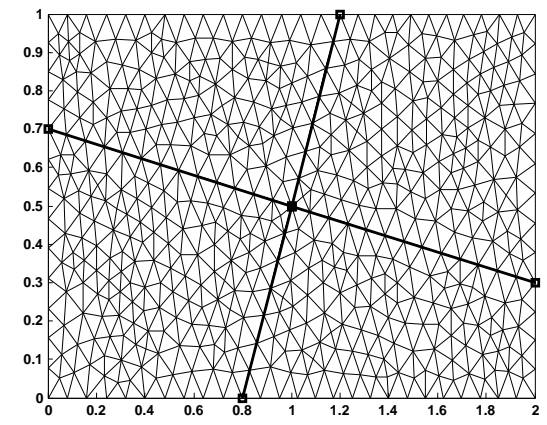

Fiøure 10: The mesh
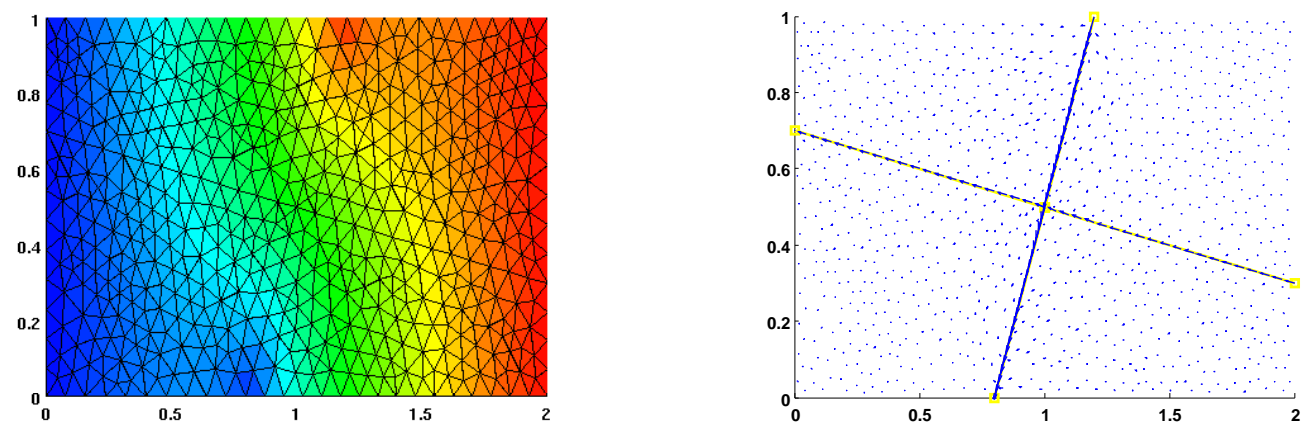

Figure 11: The numerical pressure (left) and the numerical velocity (right) 


\section{Nonconforming meshes}

In many applications one may want to use nonconforming meshes. For simplicity, in this section, we suppose that the domain is divided into only two subdomains separated by a single fracture. In the numerical discretization, the domain will be decomposed into nonoverlapping subdomains with the fracture as the interface between the two subdomains. The grids will be defined independently in each subdomain and in the fracture, see Figure 12 .
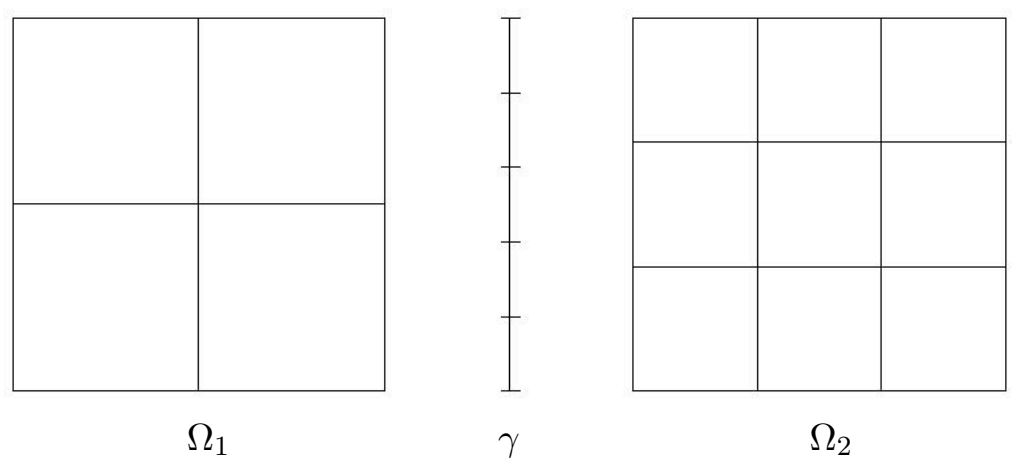

Figure 12: An example of nonconforming grids for the fracture and the subdomains

Let $\mathcal{T}_{h, i}$ be a conforming finite element partition of $\Omega_{i}, i=1,2$, and let $\mathcal{T}_{h, \gamma}$ be a conforming finite element partition of the interface $\gamma$ in $(n-1)$-dimensions. The meshes $\mathcal{T}_{h, 1}$ and $\mathcal{T}_{h, 2}$ need not form a conforming mesh on $\Omega$ and $\mathcal{T}_{h, \gamma}$ is independent of $\mathcal{T}_{h, 1}$ and of $\mathcal{T}_{h, 2}$. The notation $\mathcal{T}_{h, \gamma, i}, i=1,2$, will be used for the partition induced on $\gamma$ by the partition $\mathcal{T}_{h, i}$ on $\Omega_{i}$; i. e. $\mathcal{T}_{h, \gamma, i}, i=1,2$ consists of all of the (n-1) dimensional faces of elements of $\mathcal{T}_{h, i}$ which lie on $\gamma$. See Figure 13 .

As above, we use the lowest order Raviart-Thomas-Nedelec mixed finite element spaces associated with $\mathcal{T}_{h, i}, i=1,2$, and $\mathcal{T}_{h, \gamma}$ so that the vector variable is approximated in a space $\mathbf{W}_{\mathbf{h}}=\mathbf{W}_{\mathbf{h}, \mathbf{1}} \times \mathbf{W}_{\mathbf{h}, \mathbf{2}} \times \mathbf{W}_{\mathbf{h}, \gamma}$ and the spaces of piecewise constants associated with $\mathcal{T}_{h, i}, i=1,2$, and $\mathcal{T}_{h, \gamma}$ so that the scalar variable is approximated in a space $M_{h}=M_{h, 1} \times M_{h, 2} \times M_{h, \gamma}$.

Also as above, domain decomposition techniques are used to reduce the discrete problem to a problem posed only on the interface $\gamma$, but here we can not use directly the operator $\mathcal{S}_{i}: M_{h, \gamma} \longrightarrow M_{h, \gamma}$ as defined in Section 5.1 because of the incompatibility between the grids. Letting $M_{h, \gamma, i}$ denote the space of piecewise constants associated with $\mathcal{T}_{h, \gamma, i}, i=1,2$, we will, with some slight abuse of notation, now denote by $\mathcal{S}_{i}$ the mapping $\mathcal{S}_{i}: M_{h, \gamma, i} \longrightarrow M_{h, \gamma, i}$ defined in the obvious manner. Then, let $\mathcal{R}_{i}: M_{h, \gamma} \longrightarrow M_{h, \gamma, i}$ be the projection operator defined by

$$
\begin{aligned}
\mathcal{R}_{i}: M_{h, \gamma} & \longrightarrow M_{h, \gamma, i} \\
r_{h, \gamma} & \mapsto r_{h, \gamma, i}
\end{aligned}
$$

where

$$
r_{h, \gamma, i \mid E}=\frac{1}{|E|} \int_{E} r_{h, \gamma} \quad \forall E \in \mathcal{T}_{h, \gamma, i}
$$



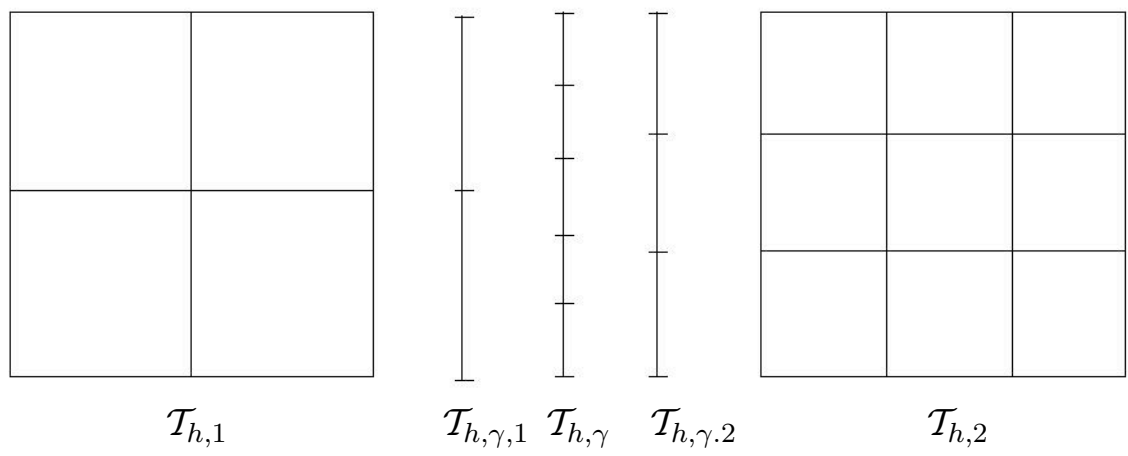

$\mathcal{T}_{h, 2}$

Figure 13: An example of a numerical discretization with nonconforming grids for the fracture and the subdomains

So, for the case of nonmatching grids the Steklov-Poincaré operator $\mathcal{S}_{i}: M_{h, \gamma} \longrightarrow M_{h, \gamma}$ of Section 5.1 is replaced by the operator $\tilde{\mathcal{S}}_{i}: M_{h, \gamma} \longrightarrow M_{h, \gamma}$ with

$$
\tilde{\mathcal{S}}_{i}=\mathcal{R}_{i}^{t} \circ \mathcal{S}_{i} \circ \mathcal{R}_{i}
$$

where $\mathcal{R}_{i}^{t}$ is just the transpose of the projection operator $\mathcal{R}_{i}$.

In the numerical experiments below, no mortar elements were introduced as there already $(n-1)$-D elements on the fracture interfaces, nor were the usual size restrictions associated with mortar elements, c.f. [4], [5] and [19], respected. We have been shown in [14] that this is sufficient in the linear case, i. e. in the case of Darcy flow in the fractures for the model of [17].

Remark: In the case of intersecting fractures in a 3-D domain, one would need either to have compatible meshes at the intersection $T$ or to use mortar elements on $T$.

\subsection{A numerical experiment for nonconforming meshes}

In this experiment the domain $\Omega \subset \mathbb{R}^{2}$ is divided into 4 subdomains $\Omega_{i}, i=1, \ldots, 4$ by 5 fracture interfaces $\gamma_{k}, k=1, \ldots 5$. The fractures $\gamma_{1}, \gamma_{2}$, and $\gamma_{3}$ intersect at a point $T_{1}$ and the fractures $\gamma_{3}, \gamma_{4}$, and $\gamma_{5}$ intersect at a point $T_{2}$, see Figure 14

The width $d_{\gamma_{k}}$ is the same for all the fractures and is equal to 0.01. The permeability in each of the subdomains is assumed to be constant and equal to $10^{-9}$, and the permeability in each fracture is assumed to be much higher and also constant: $K_{\gamma_{k}}=10^{-6}$ for each $k$. The Forchheimer coefficient $b_{\gamma_{k}}$ is taken to be 10 in all of the fractures. The upper and lower exterior boundaries of the subdomains are assumed to be impermeable, and there is a pressure drop from right to left of $10^{6}$. A pressure of $10^{6}$ is imposed at the exterior ends of $\gamma_{4}$ and $\gamma_{5}$ and a pressure of 0 is imposed on the exterior ends of $\gamma_{1}$ and $\gamma_{2}$. 


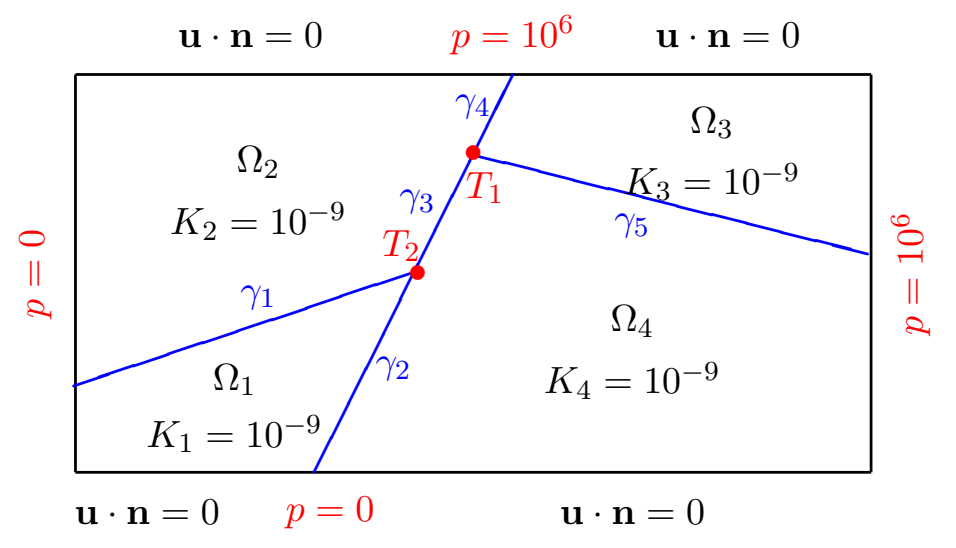

Figure 14: Test-case

The nonconforming mesh used for this test case is shown in Figure 15. It is made up of independently chosen grids for the subdomains and grids for the fracture interfaces. Here for simplicity each of the one dimensional grids is a uniform grid. The mesh parameters for the two dimensional subdomain grids and for the one dimensional fracture interface grids are given in the tables below, where by mesh parameter of a two or three dimensional grid is meant the maximum length of an edge of an element of the mesh and of a one dimensional grid the maximum length of a segment of the mesh.

- Mesh parameters for the subdomain girds

\begin{tabular}{|c||c|c|c|c|}
\hline subdomain & $\Omega_{1}$ & $\Omega_{2}$ & $\Omega_{3}$ & $\Omega_{4}$ \\
\hline mesh parameter & 0.20 & 0.10 & 0.08 & 0.05 \\
\hline
\end{tabular}

- Mesh parameters for the fracture interface grids

\begin{tabular}{|c||c|c|c|c|c|}
\hline interface & $\gamma_{1}$ & $\gamma_{2}$ & $\gamma_{3}$ & $\gamma_{4}$ & $\gamma_{5}$ \\
\hline mesh parameter & 0.035 & 0.029 & 0.07 & 0.048 & 0.11 \\
\hline
\end{tabular}

The numerical solution obtained with the mesh shown in Figure 15 is given in Figure 16 , where the approximate pressure is shown on the left and the approximate velocity field on the right. A good solution was obtained in spite of the nonconforming grid, even through no additional mortar elements were introduced. 


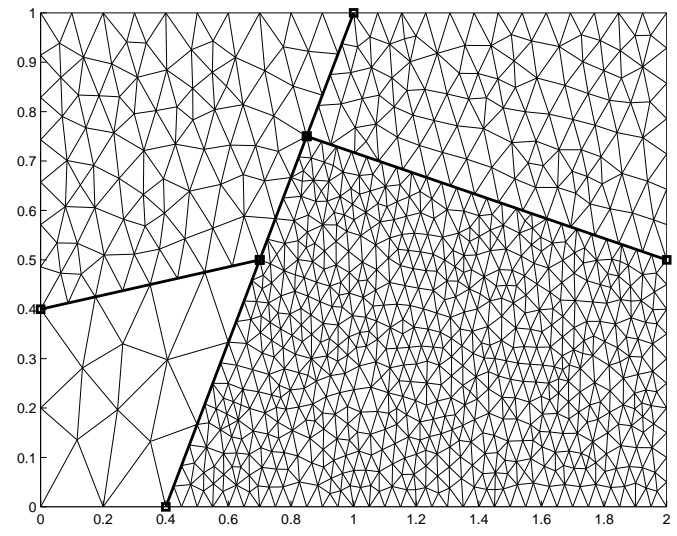

Figure 15: The nonconforming meshes
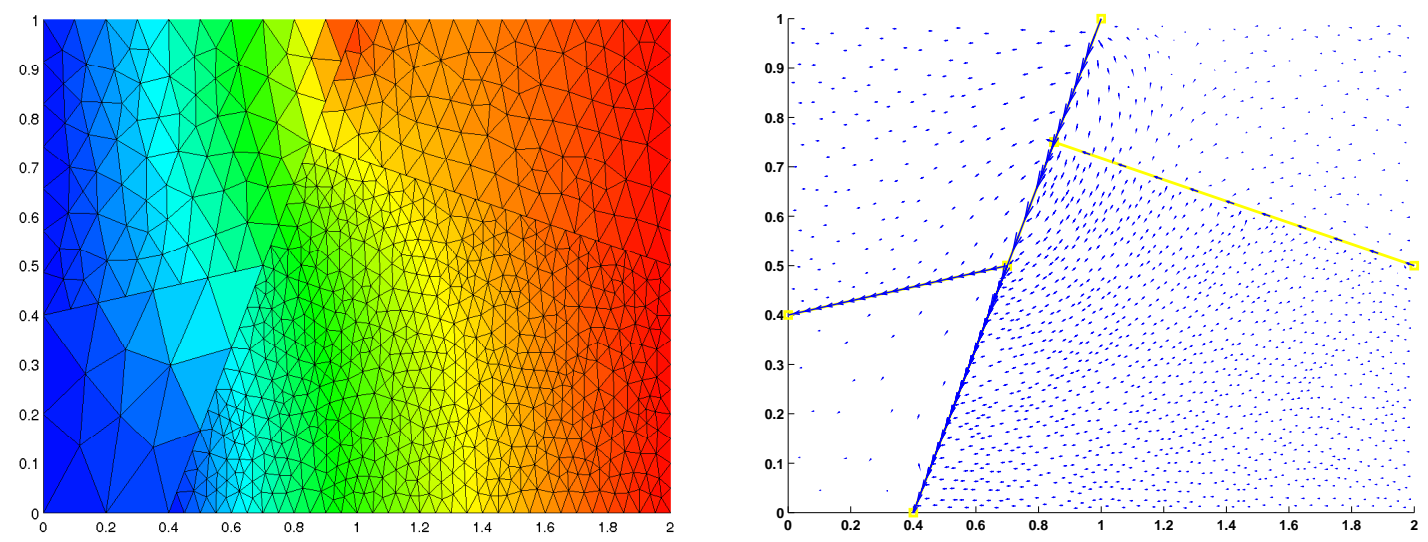

Figure 16: The pressure (left) and the velocity (right) computed solutions

\section{Conclusion}

This article is concerned with a numerical model, introduced in the short article [13], for flow in a porous medium with fractures. For this model it was assumed that the flow rate in the fractures is large enough to make it appropriate to use Forchheimer's law for modeling the flow in the fractures even though the flow in the surrounding domain is such that Darcy's law is adequate. The fractures were treated as interfaces between subdomains and nonlocal, nonlinear transmission conditions were imposed on the interfaces. Then domain decomposition techniques were used to reduce the problem to a problem posed on the interfaces. 
In this article this model is described in more detail and numerical studies are given. The model is extended to the case of intersecting fractures and to the case of nonconforming meshes.

\section{Aknowledgments}

The authors would like to thank Rachida Bouhlila and Jérome Jaffré for helpful conversations concerning this article.

\section{References}

[1] C. Alboin, J. Jaffrée, And J. E. Roberts, Domain decomposition for flow in fractured porous media, Domain Decomposition Methods in Sciences and Engineering, (1999), pp. $365-373$.

[2] L. Amir, M. Kern, V. Martin, and J. E. Roberts, Décomposition de domaine pour un milieu poreux fracturé: un modèle en 3d avec fractures qui s'intersectent, ARIMA, 5 (2006), pp. 11-25.

[3] P. Angot, T. Gallouet, And R. Herbin, Convergence of finite volume method on general meshes for non smooth solution of elliptic porblems with cracks, Proc. 2nd Int. Symposium Finite Volumes for Complex Applications, (1999), pp. 215-222.

[4] T. Arbogast, L. C. Cowsar, M. F. Wheeler, and I. Yotov, Mixed finite element methods on nonmatching multiblock grids, Siam J. Math. Anal., 37 (2000), pp. 1295-1315.

[5] C. Bernardi, Y. Maday, And A. T. Patera, A new nonconforming approach to domain decomposition: the mortar element method, Nonlinear Partial Differential Equations and Their Applications, 184 (1994), pp. 13-51.

[6] J. Douglas, T. Arbogast, and U. Hornung, Derivation of the double porosity model of single phase flow via homogenization theory, SIAM J. Math. Anal, 21 (1990), pp. 823836.

[7] J. Douglas, P. J. S. P. Leme, and T. Giorgi, Generalized Forchheimer flow in porous media, in boundary value problems for partial differential equations and applications, Research Notes in Applied Mathematics, 29 (1993), pp. 99-113.

[8] J. ERhel And J. Dreuzy, Efficient algorithms for the determination of the connected fracture network and the solution of the steady-state flow equation in fracture networks, Computers and Geosciences, 29 (2002), pp. 107-111.

[9] A. Q. ET A. Valli, Domain Decomposition Methods for partial differential equations, Claderon Press, Oxford, 1999.

[10] E. Flauraud, Méthodes de décomposition de domaines pour les écoulements en milieux poreux faillés, PhD thesis, Université Pierre et Marie Curie, 2004.

[11] P. Forchheimer, Wasserbewegung durch boden, Zeits. V. deutsch. Ing., 45 (1975), pp. $1782-1788$. 
[12] M. Fourar And M. Panfilov, Physical splitting of nonlinear effects in high-velocity stable flow through porous media, Advances in Water Resourses, 29 (2006), pp. 30-41.

[13] N. Frih, J. E. Roberts, And A. SaÂDa, Un modèle Darcy-Forchheimer pour un écoulement dans un milieu poreux fracturé, ARIMA, 5 (2006), pp. 129-145.

[14] — Modeling fractures as interfaces: a model for forchheimer fractures, à parître dans Computational Geosciences, (2008).

[15] T. Giongi, Derivation of the forchheimer law via matched asymptotic expansions, Tranport in Porous Media, 29 (1997), pp. 191-206.

[16] J. J. Douglas and T. Arbogast, Dual porosity models for flow in naturally fractured reservoirs, in Dynamics of Fluids in Hierarchial Porous Formations, (1990), pp. 77-221.

[17] V. Martin, J. Jaffré, and J. E. Roberts, Modeling fractures and barriers as interfaces for flow in porous media, SIAM Journal on Scientific Computing, 26 (2005), pp. 1667-1691.

[18] V. Reichenberger, H. Jakobs, P. Bastian, and R. Helmig, A mixed-dimensional finite volume method for multiphase flow in fractured porous media, Advances in Water Resources, 29 (2006), pp. 1020-1036.

[19] M. F. Wheeler And I. Yotov, Multigrid on the interface for mortar mixed finite element methods for elliptic problems, Computer methods in applied mechanics and engineering, 184 (2000), pp. 287-302.

[20] S. WiTAKeR, The forchheimer equation: theoretical development, Transport in Porous media, 25 (1996), pp. 27-61. 


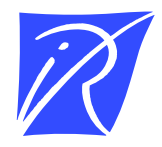

Unité de recherche INRIA Rocquencourt

Domaine de Voluceau - Rocquencourt - BP 105 - 78153 Le Chesnay Cedex (France)

Unité de recherche INRIA Futurs : Parc Club Orsay Université - ZAC des Vignes

4, rue Jacques Monod - 91893 ORSAY Cedex (France)

Unité de recherche INRIA Lorraine : LORIA, Technopôle de Nancy-Brabois - Campus scientifique

615, rue du Jardin Botanique - BP 101 - 54602 Villers-lès-Nancy Cedex (France)

Unité de recherche INRIA Rennes : IRISA, Campus universitaire de Beaulieu - 35042 Rennes Cedex (France)

Unité de recherche INRIA Rhône-Alpes : 655, avenue de l'Europe - 38334 Montbonnot Saint-Ismier (France)

Unité de recherche INRIA Sophia Antipolis : 2004, route des Lucioles - BP 93 - 06902 Sophia Antipolis Cedex (France) 